\title{
VIOLENT RELAXATION AND MIXING IN 1-D GRAVITATIONAL SYSTEMS
}

\author{
Marc Luwel \\ Vrije Universiteit Brussel \\ Brussels, Belgium
}

\section{INTRODUCTION}

The one dimensional gravitational model consists of $\mathrm{N}$ mass sheets with surface density $m_{i}$, parallel to the $(y, z)$-plane and constrained to move along the $x$-axis under influence of their mutual gravitational force $F_{i j}=-2 \pi G m_{i} m_{j} \operatorname{sgn}\left(x_{i}-x_{j}\right)$. In order to study the evolution of this one-dimensional system, the $\mathbf{N}$ Newtonian equations of motion are integrated numerically, using an "exact" double precision algorithm.

\section{STATIONARY WATERBAG CONFIGURATION}

A one dimensional gravitational system in a stationary waterbag configuration occupies a region in the two dimensional phase-space with a constant phase density, enclosed by a single energy contour. Such a system, with a bimodal mass distribution, containing $N_{\ell}$ light particles with mass $m_{\ell}$ and $N_{h}$ heavy particles with mass $m_{h}$, is studied. In order to follow its macroscopic evolution, the equipartition ratio $k(t)$. defined as the ratio of the average kinetic energies of the light and the heavy species, is measured during the integration (Luwel and Severne 1985).

For a system in a stationary waterbag configuration with both mass groups initially well mixed, no violent relaxation is observed. Moreover, the mixing ratio, which initially has its well mixed value $k(t=0)=m_{\ell} / m_{h}$, shows no evolution towards equipartition $\left(k_{e q}=1\right)$ on a timescale of order $N t_{d}$, where $t_{d}$ is the crossing or dynamical timescale (Figure 1 ).

In systems initially in a stationary waterbag configuration, two particle effects turn out to be inoperative, at least on a timescale of order $N t_{d}$ (Figure 2). Consequently, in the absence of any macroscopic evolution in such a system, one would expect that each particle remains in its motion close to a stationary phase space trajectory of nearly constant energy. But plots of the particle trajectories and of the evolution of the individual particle energies show that under influence of mean field fluctuations the particles migrate slowly through large energy intervals, on a timescale $t_{m i x}$ of order $10 t_{d}$ (Figure 2). 


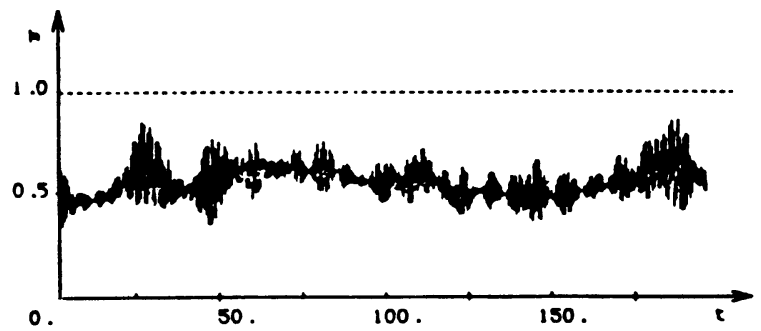

Figure 1. Evolution of the equipartition ratio $k(t)$ for system I containing $N_{\ell}=100$ light particles with mass $m_{\ell}=1 / 200$ and $N_{h}=50$ heavy particles with mass $m_{h}=2 m_{\ell}$, and initially in a stationary waterbag configuration with both mass groups distributed uniformly.
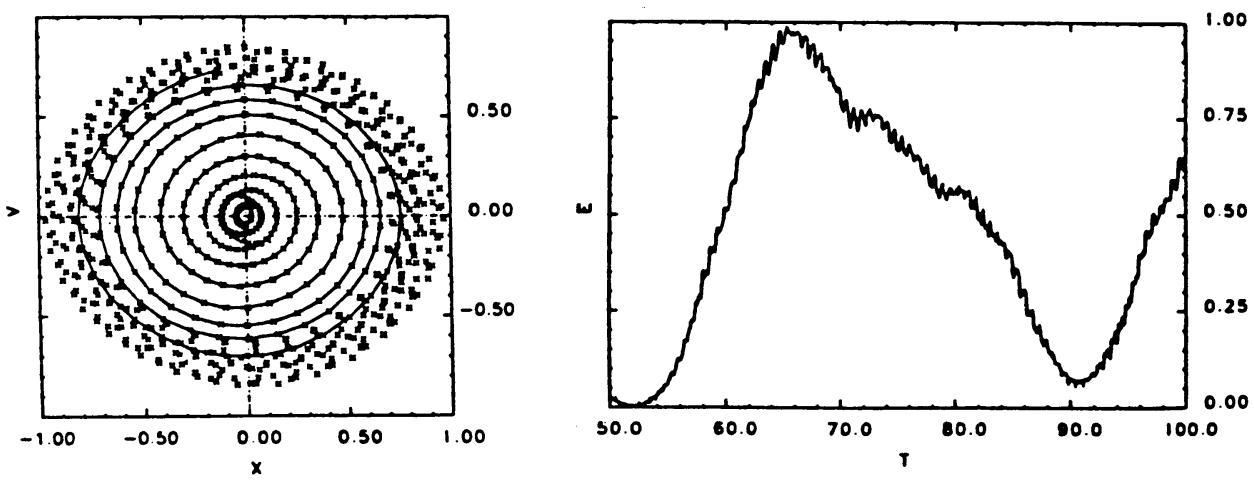

Figure 2. a) Phase orbit of a typical particle of system I. For $50 t_{d}<$ $t<80 t_{d}$ the crosses give the phase positions at successive time intervals of $0.04 t_{d}$; they are connected by a continuous line for $54 t_{d}<t<65 t_{d}$.

b) Evolution of the energy of the same particle.

\section{CONCLUSIONS}

This hitherto unobserved mixing process, which becomes clearly evident due to the inefficiency of two particle effects in systems initially in a stationary waterbag configuration, is also observed as an evolution phase intermediate between the violent and the collisional phase in systems with less peculiar initial conditions. Moreover, this mixing process turns out to be the missing dynamical element of Shu's theory of violent relaxation (Shu 1978). Numerical experiments on systems characterized by two phase densities show that, after an initial evolution towards the LyndenBell distribution, these systems start evolving towards the Shu distribution until discrete particle effects become dominant (Severne and Luwel 1986).

\section{REFERENCES}

Luwel, M. and Severne, G., 1986 to be published.

Severne, G. and Luwel, M., 1985 Astron. Astroph., 152, 305.

Shu, F., 1978 Astroph. J., 225, 83. 